\title{
Initial steps of base excision repair on DNA-substrates with non-canonical structures
}

\author{
Alexandra A. Kuznetsova \\ Institute of Chemical Biology and Fundamental Medicine \\ Siberian Branch of Russian Academy of Sciences \\ Novosibirsk, Russia \\ sandra-k@niboch.nsc.ru \\ Olga S. Fedorova \\ Institute of Chemical Biology and Fundamental Medicine \\ Siberian Branch of Russian Academy of Sciences \\ Novosibirsk, Russia \\ fedorova@niboch.nsc.ru
}

Anastasiia T. Davletgildeeva

Institute of Chemical Biology and Fundamental Medicine Siberian Branch of Russian Academy of Sciences

Novosibirsk, Russia

davleta94@gmail.com

Nikita A. Kuznetsov

Institute of Chemical Biology and Fundamental Medicine Siberian Branch of Russian Academy of Sciences Novosibirsk, Russia

Nikita.Kuznetsov@niboch.nsc.ru

\begin{abstract}
Although canonical B-form is thought to be prevalent in human genome, DNA is able to adopt many noncanonical forms. Such DNA sites are often localized in genome regions having important biological significance. For example, human telomeres contain repeated TTAGGG elements, in which the 3 '-exposed strand may adopt a Gquadruplex structure. In addition, more than $40 \%$ of human genes have been found to contain at least one sequence near the promoter regions, which potentially could form a quadruplex structure. Some regions of such nature have an increased susceptibility to oxidative damage. Indeed the guanine-rich regions of telomeres are hotspots for oxidation forming 8-oxoguanine, thymine glycol as well as abasic sites (AP-sites), the lesions that are handled by the base excision repair (BER) pathway. However, the features of DNA repair processes in non-canonical structures, including quadruplexes, are still poorly understood. Therefore, the purpose of this work was a comparative analysis of the efficiency of the removal of damaged nucleotides from noncanonical DNA structures by human BER enzymes. We designed a set of DNA substrates such as DNA bubbles, DNA loops and G-quadruplexes. We analyzed activity of DNA glycosylases and AP endonuclease on model substrates. Direct detection of the product formation by PAGE allowed to estimate the efficiency of enzymatic hydrolysis of damaged Gquadruplexes under the same experimental conditions. The stages of formation of enzyme-substrate complexes and catalysis were analyzed by stopped-flow technique, which allows to register conformational rearrangements of model DNA substrates.
\end{abstract}

Keywords - Base excision repair, DNA glycosylases, AP endonuclease, DNA non-canonical structures

Human genome DNA adopts canonical B-form righthanded double-helical structure. However, many alternative DNA conformations are known to exist, and their roles in affecting biological processes have just begun to be understood. In particular, tandem DNA repeats for example, GGG-rich sequences, which are found in telomeric DNA, known to play critical role in aging and disease development. In addition, more than $40 \%$ of human genes have been found to contain at least one sequence near the promoter regions, which potentially could form a quadruplex structure. Moreover, complex nucleic acid structures can be formed during the processes of DNA replication and transcription, and other cellular events.

These domains of the genome could be susceptible to oxidative attack and other endogenous and exogenous agents such as highly reactive cell metabolites, external environmental compounds, UV or ionizing irradiation. Abasic sites are estimated to arise spontaneously $\sim 10,000$ times per mammalian genome equivalent per day. However, the features of DNA repair processes in non-canonical structures, including DNA bubbles, DNA loops or Gquadruplexes, are still poorly understood.

It is known that recognition and removal of non-bulky damage of nitrogenous bases proceed via the base excision repair (BER) pathway. In general, the BER pathway includes sequential actions of two enzymes for DNA incision: a DNA glycosylase and an AP endonuclease. DNA glycosylases generate AP-sites and blocking 3'-end groups, which should be removed in subsequent steps of BER in order to initiate the DNA repair synthesis and ligation. An AP endonuclease hydrolyses the phosphodiester bond located $5^{\prime}$ to the AP-site and introduces a break into the deoxyribophosphate backbone, in addition, it removes the remaining 3'-blocking groups: either the 3 '-phospho- $\alpha, \beta$ unsaturated aldehyde or the $3^{\prime}$-terminal phosphate group.

Therefore, the purpose of this work was a comparative analysis of the efficiency of the removal of damaged nucleotides from DNA non-canonical structures by human BER enzymes. We analyzed activity and substrate specificity of 8-oxoguanine-DNA glycosylase (OGG1), endonuclease VIII-like 1 (NEIL1), endonuclease III (NTH1) and AP endonuclease (APE1) to their specific damaged nucleotides. As the control prokaryotic formamidopyrimidine-DNA glycosylase (Fpg) and endonuclease VIII (Nei) were used. The model Gquadruplexes contained 8-oxoguanine (oxoG), thymine glycol (Tg) or stable AP-site analog (F-site) at the loop and core region were used. A set of DNA duplexes containing in the central part $1-5 \mathrm{nt}$ loops with damaged nucleotide served as bulge-substrates. Also control G-quadruplex without damage and control duplexes with Tg, oxoG and Fsite were used for comparison of cleavage activity. In order to obtain structural insights, we first analyzed the ODNs by CD spectroscopy, under several buffer conditions including those required for the enzymatic assays.

Direct detection of the product formation by PAGE allowed to estimate the efficiency of enzymatic hydrolysis of damaged DNA under the same experimental conditions. The model 17-nt duplexes containing a oxoG, Tg or F-site were used in order to compare DNA glycosylases and AP endonuclease activity relative to damaged G-quadruplexes and bulge-substrates. In the control DNA duplexes APE1 
makes an incision of the phosphodiester bond on the 5 '-side of F-site, Fpg, Nei and NEIL1 lead to formation of the reaction product corresponding to the $\beta, \gamma$-elimination, while OGG1 and NTH1 lead to formation of the reaction product corresponding to the $\beta$-elimination. Analysis of product accumulation using gel-electrophoresis has shown that APE1 excises all of the DNA substrates used, i.e. DNA quadruplexes, containing the single F-site in core or loop regions and bulged DNA duplexes, containing the F-site. The interaction of Fpg with Q4-oxoG and Nei and NEIL1 with Q4-Tg led the formation of cleavage product. It should be noted that cleavage of damaged quadruplex Q4-Tg by NTH1 was inefficient. Interaction of OGG1 with Q4-oxoG did not lead to the substrate cleavage at all.

The stages of formation of enzyme-substrate complexes and catalysis were analyzed by stopped-flow technique, which allowed to register conformational rearrangements of model DNA substrates. DNA substrates were labeled by $\mathrm{aPu}$ or FAM/BHQ1. It should be noted that we used two type of 17-nt duplex substrates: the first one contained FAM and BHQ1 residues on the opposite side of the duplex, whereas in the second one the FAM and BHQ1 residues were situated at the one side of the duplex. These substrates allow to register not only the substrate cleavage but also DNA bending and local melting in the course of catalytic complex formation.

Taking together, a set of DNA substrates with noncanonical structures was tested to evaluate the structural aberrations in non-canonical structures required for the cleavage of damaged DNA by human DNA glycosylases and AP endonuclease.

\section{ACKNOWLEDGMENT}

The part of this work involving analysis of DNA glycosylases was specifically funded by Russian Foundation of Basic Research grant 19-04-00012. The part of this work involving analysis of AP endonuclease was specifically funded by Russian Science Foundation grant 18-14-00135. 